\title{
Burnout y afrontamiento en profesionales de la salud
}

\section{Burnout and coping in health professionals}

\author{
Maricela Osorio-Guzmán, Carlos Prado-Romero \\ y Georgina E. Bazán-Riverón
}

\section{Universidad Nacional Autónoma de México ${ }^{1}$}

Autor para correspondencia: Maricela Osorio-Guzmán,mosorio@unam.mx.

\section{RESUMEN}

\begin{abstract}
El objetivo de este trabajo fue analizar la correlación entre el nivel de burnout y el tipo de afrontamiento en una muestra de profesionales de la salud. Participaron 60 profesionistas, a los que se les aplicaron dos instrumentos: el Maslach Burnout Inventory-Human Service Survey (MBI-HSS) y el Cuestionario de Afrontamiento al Estrés (CAE). De acuerdo con las áreas evaluadas con el MBI-HSS, se encontró que $79 \%$ de los participantes mostraron bajo cansancio emocional; $64 \%$ obtuvieron puntuaciones bajas en despersonalización, y $71 \%$ una alta realización profesional. Los datos del CAE revelaron que las estrategias de afrontamiento más utilizadas fueron de tipo racional. Hubo correlaciones positivas significativas entre diferentes áreas de los instrumentos. El síndrome de burnout se manifestó en la muestra en un nivel bajo, siendo la estrategia de afrontamiento más utilizada la solución de problemas.
\end{abstract}

Palabras clave: Burnout; Adaptación psicológica; Estrés psicológico; Cuerpo médico de hospitales.

\begin{abstract}
The objective of the present study was to analyze the correlation between level of burnout and type of coping in a sample of health professionals. A total of 60 healthcare professionals participated, and completed the Maslach Burnout Inventory-Human Service Survey (MBI-HSS), and the Stress Coping Questionnaire (SCQ). Regarding the areas evaluated with the MBS-HSS, 79\% of the participants scored low in emotional fatigue, 64\% obtained low scores in depersonalization, and $71 \%$ had high professional realization. The SCQ data revealed that the coping strategies most used were of rational type. Significant positive correlations were also found among different areas of the instruments. The burnout syndrome showed low levels in the sample, and the most commonly used coping strategy was problem solving.
\end{abstract}

Keywords: Burnout; Psychological adaptation; Psychological stress; Hospital medical corps.

Recibido: $31 / 01 / 2020$

Aceptado: 30/05/2020

${ }^{1}$ Facultad de Estudios Profesionales Iztacala, Av. de los Barrios 1, 54090 Los Reyes Iztacala, Tlalnepantla, Estado de México, México, tel. (55)56-23-13-33, ext. 39806, Maricela Osorio-Guzmán http://orcid.org/0000-0001-7798-5301, Carlos Prado https://orcid.org/0000-0003-08090672 y Georgina Bazán https://orcid.org/0000-0001-6582-6370. correos electrónicos: mosorio@unam.mx, carlosprador9318@gmail.com y gebrmx@yahoo.com.mx. 
$\mathrm{E}$ 1 concepto de burnout, según Carlín y Garcés (2010), surge en 1974 a partir de las observaciones de Freudenberger mientras trabajaba como voluntario en la clínica para toxicómanos Free Clinic de Nueva York, en la que, pasado un periodo que iba de uno a tres años, sus compañeros sufrían una pérdida progresiva de energía y motivación en su labor, acompañada de claros síntomas de agotamiento, ansiedad y depresión.

El burnout es un síndrome que consta de tres dimensiones: el cansancio emocional (falta de recursos emocionales y sensación de no poder ofrecer nada a nivel afectivo), la despersonalización (serie de actitudes inhumanas, negativas, frías, cínicas y duras hacia los beneficiarios de su trabajo) y la baja realización profesional (sentimiento de inadecuación personal o falta de logros personales y profesionales) (Feitosa, Sousa, Alves, Gómez y Méndez, 2016; Maslach, Jackson y Leiter, 1996).

Este síndrome es el resultado de la acumulación de estrés en el ambiente laboral, y cuando es crónico puede tener efectos perjudiciales para la salud física, psicológica y emocional, lo que trae consigo diversos problemas, como apatía a la calidad clínica, ansiedad, depresión, ausentismo, agresividad y aumento del consumo de sustancias como café, alcohol, barbitúricos, alimentos y tabaco, y comprometiendo incluso las relaciones familiares (Díaz, 2010).

Hay diversos factores que aumentan la probabilidad de que aparezca dicho síndrome, como el deseo persistente de marcar una diferencia con los demás y obtener resultados excepcionales; tener un trabajo muy comprometido con el dolor y el sufrimiento; establecer relaciones negativas y antagonistas con los colegas; mostrar una elevada autoexigencia; mostrar una baja tolerancia al fracaso; tener necesidad de controlar a los demás; manifestar un sentimiento de omnipotencia ante una tarea, o sentir que se es indispensable ("nadie puede hacerlo más que yo") (De la Rosa et al., 2015; Rodríguez, Zarco y Gonzáles, 2009). Generalmente, tal proceso de evolución del síndrome comienza con un exceso de carga laboral, real o percibida, y estrés, que si no se trata oportunamente puede desencadenar problemas de salud, $y$ en casos extremos hasta el suicidio (Moreno,
Seminotti, Garrosa, Rodríguez y Morante, 2005; Weber y Jaekel, 2000).

Uno de los instrumentos más utilizados para medir las implicaciones y el grado de afectación debidos al burnout en el personal de salud es el Maslach Burnout Inventory-Human Services Survey (MBI-HSS en lo sucesivo), con el cual se ha desarrollado un sinnúmero de investigaciones en diferentes áreas de intervención de las ciencias biomédicas y psicológicas. Entre los hallazgos más importantes realizados en el área de la salud están los siguientes: 1) El grado de burnout en los profesionales de la salud está correlacionado negativamente con la experiencia laboral (Álvarez, Arc, Barrios y Sánchez, 2005; Pereda, Márquez, Hoyos y Yáñez, 2009; Castillo, Orozco, y Alvis, 2015; Moreno et al., 2005); 2) El sexo femenino muestra mayores niveles de cansancio emocional, mientras que el masculino presenta niveles más elevados de despersonalización (Aranda, 2006; Dickinson et al., 2007; Martín, Hernández, Arnillas y García, 2009); 3) Los trabajadores del área de la salud suelen habituarse al estrés constante (Ballinas, Alarcón y Balseiro, 2009), y 4) La actividad médica y la percepción de la alta relevancia del trabajo generan una adecuada satisfacción laboral (Balcázar, Montejo y Ramírez 2015; Pistelli, Perochena, Moscoloni y Tarrés, 2011).

Las personas hacen frente a las situaciones de estrés de distintas formas, ya sea eliminando la fuente, o bien actuando sobre sus propios pensamientos, emociones y reacciones psicosomáticas para reducir su efecto (Manassero, Vázquez, Ferrer, Fornés y Fernández, 2003; Parrello, Ambrosetti, Iorio y Castelli, 2019), a lo que se le denomina comúnmente afrontamiento. Este es definido como "los esfuerzos cognitivos y conductuales constantemente cambiantes que se desarrollan para manejar las demandas específicas externas o internas que exceden o desbordan los recursos del individuo" (Lazarus y Folkman, 1984). Se distinguen dos principales tipos: el enfocado en la solución de problemas al actuar sobre el ambiente o sobre uno mismo para alterar la fuente estresante (Sandín y Chorot, 2003), y el enfocado en la regulación emocional, o sea, disminuyendo el efecto psicológico que causan las situaciones estresantes 
(Acosta, Chaparro y Rey, 2008; Guerrero y Vicente, 2001).

Así, las estrategias de afrontamiento operan para disminuir el efecto estresante generado por la amenaza, y cuanto más lo reducen, puede hablarse de un afrontamiento más efectivo; también la forma en que se afrontan tales episodios tiene un efecto en el funcionamiento psicosocial, esto es, que un afrontamiento eficaz puede hacer que las personas tengan un mejor funcionamiento social, no obstante el número de episodios de estrés (Galán y Camacho, 2012).

Hay numerosas investigaciones que relacionan el burnout con el afrontamiento, cuyos principales resultados muestran que los profesionales de la salud tienden regularmente a llevar a cabo afrontamientos de tipo racional, mientras que la estrategia de recurrir a la religión es la menos utilizada (Piñeiro, 2013); así como que los varones emplean sobre todo el afrontamiento de evitación (Paris y Omar, 2009), y que el síndrome de burnout y las estrategias de afrontamiento están correlacionadas significativamente en los profesionales de la salud.

De lo anterior se desprende que las estrategias de afrontamiento pueden modificar de un modo importante la forma en que se experimenta el estrés al hacer que los efectos del burnout disminuyan.

Por consiguiente, el objetivo del presente estudio fue analizar la correlación entre los niveles de burnout y los tipos de afrontamiento de una muestra de profesionales de la salud.

\section{MÉTODO}

\section{Participantes}

Participaron 60 profesionales de la salud, hombres y mujeres, de veintiún estados de la República Mexicana, con edades comprendidas entre 21 y 75 años, y con una antigüedad laboral de 1 a 45 años. La selección se realizó a partir de un muestreo incidental. El diseño utilizado fue de tipo transversal y correlacional (Shaughnessy, Zechmeister y Zechmeister, 2007).

\section{Instrumentos}

En lo referente a los instrumentos de evaluación, se recolectaron los datos sociodemográficos de los participantes e información general sobre su ejercicio profesional.

Maslach Burnout Inventory-Human Service Survey (MBI-HSS) (Maslach y Jackson, 1981).

Compuesto por 22 reactivos distribuidos en los tres factores mencionados, los que se valoran en una escala Likert de seis opciones; puntuaciones altas en las dos primeras escalas y baja en la tercera indican la presencia del síndrome. Las consistencias internas de sus escalas, medidas por el coeficiente alfa de Cronbach, son las siguientes: Cansancio emocional, 0.86; Despersonalización, 0.81, y Realización profesional, 0.53 (Meda, Moreno, Rodríguez, Morante y Ortiz, 2008).

\section{Cuestionario de Afrontamiento del Estrés (CAE)} (Chorot y Sandín, 1987).

Consta de 42 reactivos que se evalúan en una escala tipo Likert de cinco opciones de respuesta, el cual valora siete tipos de afrontamiento: focalizado en la solución de problemas, búsqueda de apoyo social, evitación, autofocalización negativa, reevaluación positiva, religión y expresión emocional abierta. La consistencia interna de este instrumento oscila entre 0.66 y 0.91 según las escalas que lo componen (González y Landero, 2007).

\section{Procedimiento}

Se identificó a los profesionales de la salud en el marco de un congreso multidisciplinario nacional especializado, a los cuales se les solicitó su participación, requiriéndoles, en caso de aceptar, la firma de un formato de consentimiento informado. Los instrumentos se aplicaron en un aula del recinto donde se llevaba a cabo el evento. Hecho lo anterior, se procedió a seleccionar aquellos instrumentos que habían sido respondidos por completo para realizar la captura de los datos y su análisis. 


\section{Análisis de datos}

Una vez revisado y aprobado el proyecto general del cual se deriva el presente trabajo por el Comité de Bioética de la Carrera de Psicología de la Facultad de Estudios Superiores Iztacala de la Universidad Nacional Autónoma de México, se efectuó el análisis descriptivo de las variables sociodemográficas de la muestra; se calcularon y describieron los puntajes de cada una de las escalas que componen los instrumentos; se compararon las medias de los diversos subgrupos (profesión, institución laboral y años de ejercicio profesional) a través de un análisis de varianza de un factor con comparaciones post hoc mediante la prueba HSD de Tukey para determinar las diferencias significativas entre cada uno de estos subgrupos, y finalmente se obtuvieron las correlaciones entre las diferentes variables y las áreas del cuestionario mediante la prueba $r$ de Pearson. El análisis de datos se llevó a cabo utilizando el programa estadístico SPSS, v. 25.

\section{RESULTADOS}

Respecto a las variables sociodemográficas, se obtuvo que $69 \%$ de los participantes fueron mujeres; la media de edad fue de 43.5 años (D.E. $=11.8$ ), y en promedio tenían 16 años de experiencia profesional (D.E. $=10.64)$; respecto a la profesión de los participantes, en la Tabla 1 se muestra.

Los participantes provenían de veintiún estados de la República Mexicana, donde destacan en primer lugar la Ciudad de México (25.4\%); en segundo lugar, Jalisco y Veracruz (9.5\%); y en tercer lugar Baja California, Nuevo León, Oaxaca y Puebla (6.3\%). En la Tabla 1 se puede observar las especialidades de dichos participantes y las instituciones en donde laboraban.

Tabla 1. Profesión e institución donde laboran los participantes.

\begin{tabular}{|l|r|r|}
\hline \multicolumn{1}{|c|}{ Profesión / Especialidad } & Frec. & \% \\
\hline Enfermería & 7 & 11.1 \\
\hline Genetista & 4 & 6.3 \\
\hline Hematología & 30 & 47.6 \\
\hline Medicina General & 1 & 1.6 \\
\hline Medicina Interna & 2 & 3.2 \\
\hline Odontología & 3 & 4.8 \\
\hline Psicología & 4 & 6.3 \\
\hline Químicos & 6 & 9.5 \\
\hline Rehabilitación & 3 & 4.8 \\
\hline Trabajo Social & 1 & 1.6 \\
\hline Institución donde labora & 8 & \\
\hline Hospitales universitarios & 26 & 41.3 \\
\hline Instituto Mexicano del Seguro Social & 3 & 4.8 \\
\hline $\begin{array}{l}\text { Instituto de Seguridad Social al Servicio } \\
\text { de los Trabajadores del Estado }\end{array}$ & 5 & 7.9 \\
\hline Instituciones privadas & 19 & 30.2 \\
\hline Secretaría de Salud y Asistencia & & \\
\hline
\end{tabular}

\section{Niveles de burnout}

A partir de los datos recopilados, se observó que los participantes exhibían niveles bajos de cansan- cio emocional y despersonalización, mientras que puntuaron alto en el área de realización profesional (Tabla 2). 
Tabla 2. Niveles de las puntuaciones obtenidas en las escalas del Maslach Burnout Inventary (MIB-HSS) por los profesionales de la salud.

\begin{tabular}{|l|c|c|c|}
\hline \multicolumn{1}{|c|}{ Escala } & Bajo & Medio & Alto \\
\hline Cansancio emocional & $79 \%$ & $11 \%$ & $10 \%$ \\
\hline Despersonalización & $64 \%$ & $22 \%$ & $14 \%$ \\
\hline Realización profesional & $16 \%$ & $13 \%$ & $71 \%$ \\
\hline
\end{tabular}

Por otra parte, no se encontraron correlaciones significativas entre las escalas que componen el MIBHSS y las variables sociodemográficas analizadas; sin embargo, se encontraron diferencias significativas en los niveles de realización profesional dependiendo de la institución donde laboran los participantes $(\mathrm{F}=2.36 ; p<0.04)$; tales diferencias se hallaron entre el Instituto Mexicano del Seguro Social (IMSS) y el Instituto de Seguridad Social al Servicio de los Trabajadores del Estado (ISSSTE) $(t=2.54 ; \mathrm{gl}=27 ; p<0.02)$, obteniendo medias más altas en el primero. También hubo diferencias significativas en el área de la realización profesional y en el sexo $(t=2.6 ; \mathrm{gl}=60 ; p<0.01)$, siendo esas medias mayores en los hombres.

\section{Tipos de afrontamiento}

Referente a los tipos de afrontamiento, se encontró que las estrategias más utilizadas fueron las encaminadas a un afrontamiento racional de las situaciones, siendo las más frecuentes las focalizadas en la solución de problemas, la reevaluación positiva y la búsqueda de apoyo social (Tabla 3 ).

Tabla 3. Media y desviación estándar de las puntuaciones obtenidas en los tipos de afrontamiento indicados en el Cuestionario de Afrontamiento del Estrés (CAE).

\begin{tabular}{|l|r|c|}
\hline \multicolumn{1}{|c|}{ Escala } & \multicolumn{1}{c|}{ M } & D.E \\
\hline Focalizado en la solución del problema (FSP) & 15.57 & 4.83 \\
\hline Autofocalización negativa (AFN) & 4.62 & 3.30 \\
\hline Reevaluación positiva (REP) & 11.89 & 3.99 \\
\hline Expresión emocional abierta (EEA) & 4.19 & 3.58 \\
\hline Evitación (EVT) & 6.89 & 4.59 \\
\hline Búsqueda de apoyo social (BAS) & 8.16 & 5.03 \\
\hline Religión (RLG) & 5.38 & 5.61 \\
\hline
\end{tabular}

Por otra parte, se encontraron correlaciones bajas negativas significativas entre la expresión emocional abierta y la edad $(r=-0.35 ; p<0.01)$ y la expresión emocional abierta y los años de ejercicio profesional $(r=-0.32 ; p<0.01)$. Asimismo, se hallaron diferencias significativas, en primer término, entre la edad de los participantes y la expresión emocional abierta $(\mathrm{F}=2.44 ; p<0.00)$; la segunda entre la edad y el afrontamiento enfocado en la religión ( $\mathrm{F}=2.2 ; p<0.01)$, y finalmente entre la especialidad y el afrontamiento enfocado en la religión $(\mathrm{F}=2.2 ; p<0.03)$.

\section{Relación afrontamiento-burnout}

Se calculó la relación entre los puntajes obtenidos en el MIB-HSS y el CAE, encontrándose, por ejemplo, que hubo correlaciones positivas significativas entre el cansancio emocional y tres tipos de afrontamiento, también se observa una asociación negativa significativa con el tipo de afrontamiento focalizado en resolver el problema; en el caso de la despersonalización, se hallaron asociaciones moderadas positivas significativas con la autofocalización negativa y la expresión emocional abierta $\mathrm{y}$, por último, respecto a la realización profesional, se obtuvieron correlaciones positivas significativas con diferentes estrategias de afrontamiento (Tabla 4). 
Tabla 4. Correlaciones entre las escalas de los instrumentos MIB-HSS y CAE.

\begin{tabular}{|l|c|c|c|}
\hline \multicolumn{1}{|c|}{ Escalas } & $\begin{array}{c}\text { Cansancio } \\
\text { emocional }\end{array}$ & Despersonalización & $\begin{array}{c}\text { Realización } \\
\text { profesional }\end{array}$ \\
\hline Focalizado en la solución de problemas & $-.266^{*}$ & $-.403^{* *}$ & $.453^{* *}$ \\
\hline Reevaluación positiva & .032 & -.008 & $.388^{* *}$ \\
\hline Autofocalización negativa & $.506^{* *}$ & $.484^{* *}$ & .015 \\
\hline Expresión emocional abierta & $.397^{* *}$ & $.371^{* *}$ & .038 \\
\hline Evitación & $.303^{*}$ & .126 & .125 \\
\hline Búsqueda de apoyo social & .070 & .430 & $.442^{* *}$ \\
\hline Religión & -.001 & .003 & .226 \\
\hline
\end{tabular}

(*) La correlación es significativa al nivel 0.05

(**) La correlación es significativa al nivel 0.01

\section{DISCUSIÓN}

Como se mencionó antes, el síndrome de burnout es un fenómeno que aparece frecuentemente en personas que trabajan bajo un estrés considerable (Díaz, 2010); por ello, es necesario llevar a cabo investigaciones en diferentes muestras y ámbitos de trabajo para analizar la ocurrencia del problema y los niveles que alcanza, y con ello plantear estrategias de intervención en áreas específicas.

Respecto a las diferencias encontradas entre los grupos, se pudieron observar niveles más altos de realización profesional según la institución donde laboraban los participantes, siendo los trabajadores del IMSS quienes obtuvieron las medias más altas; lo anterior puede estar asociado a las prestaciones y condiciones laborales de las que gozan, aspecto que podría ser motivo de futuros análisis. También se observaron diferencias en las áreas de realización profesional y en el sexo, obteniéndose una media mayor en los hombres, datos que coinciden con los de otros estudios (Aranda, 2006; Dickinson et al., 2007; Martín et al., 2009).

Respecto a las asociaciones entre los tipos de afrontamiento con las variables sociodemográficas, se observa una asociación negativa entre la edad y el tiempo de ejercicio profesional con la expresión emocional abierta (la cual se evalúa con ítems como "Descargué mi mal humor con los demás"; "Insulté a ciertas personas", etc.), por lo que se infiere que la experiencia puede ser un factor que brinda herramientas a los profesionales de la salud para desarrollar un afrontamiento de tipo racional
(Álvarez et al., 2005; Dickinson et al., 2007; Martin et al., 2009).

A partir de la relación existente entre los tipos de afrontamiento y los niveles de burnout, se puede inferir que aquellas personas que muestran en mayor medida afrontamientos racionales (solución de problemas, búsqueda de apoyo social y reevaluación positiva) tienden a padecer niveles menores de cansancio emocional y despersonalización, y mayores de realización profesional -datos que coinciden con los de autores como Galán y Camacho (2012) -, en contraposición con aquellas que exhiben afrontamientos predominantemente emocionales (autofocalización negativa, religión, expresión emocional abierta y evitación).

A partir de lo anterior, se puede concluir que el síndrome de burnout estuvo presente, en algún grado, en la totalidad de la muestra analizada; aunque los niveles fuesen bajos, según varios autores, existe el riesgo (Álvarez et al., 2005; Aranda, 2006; Castillo et al., 2015; Díaz, 2010; Dickinson et al., 2007; Moreno et al., 2005; Pereda et al., 2009; Weber y Jaekel, 2000) de que estos profesionales manifiesten en el mediano y largo plazo un deterioro progresivo de sus relaciones interpersonales, cuadros de ansiedad, depresión y adicciones, así como de conductas automáticas, irritación constante, refugiándose en un lenguaje excesivamente técnico en sus interacciones con los demás.

También se encontró que el síndrome de burnout apareció en mayor grado en las mujeres y en los profesionistas que laboraban en instituciones diferentes al IMSS. 
Referente a los tipos de afrontamiento, se apreció que las estrategias más utilizadas fueron las focalizadas en la solución de problemas, la reevaluación positiva y la búsqueda de apoyo social, esto es, las encaminadas a afrontar racionalmente las situaciones estresantes; una vez identificados estos tipos de afrontamiento, es posible estructurar diversas actividades que fortalezcan tales habilidades.

Aunque los resultados de la presente investigación son interesantes, se considera que el he- cho de haber realizado el levantamiento de datos durante un congreso especializado determinó que la porción más amplia de la muestra fuera de especialistas en hematología, por lo que se sugiere para futuros trabajos incluir a un número mayor de participantes de otras especialidades.

Finalmente, como resultado de este tipo de estudio, es necesario plantear programas de prevención cuyos objetivos sean la modificación de comportamientos asociados al burnout.

Citación: Osorio G., M., Prado R., C. y Bazán R., G.E. (2021). Burnout y afrontamiento en profesionales de la salud. Psicología y Salud, 31(2), 267-274. https://doi.org/10.25009/pys.v31i2.2695.

\section{REFERENCIAS}

Acosta P., A., Chaparro L., C. y Rey C., A. (2008). Calidad de vida y estrategias de afrontamiento en pacientes con insuficiencia renal crónica sometidos a hemodiálisis, diálisis peritoneal o trasplante renal. Revista Colombiana de Psicología, 17, 9-26.

Álvarez A., M., Arc M., L., Barrios A., E. y Sánchez A., R. (2005). Síndrome de burnout en médicos de hospitales públicos de la ciudad de Corrientes. Revista de Posgrado de la VIa. Cátedra de Medicina, 141, 27-30.

Aranda, C. (2006). Diferencias por sexo, síndrome de burnout y manifestaciones clínicas, en los médicos familiares de dos instituciones de salud, Guadalajara, México. Revista Costarricense de Salud Pública, 29, 1-7.

Balcázar L., E., Montejo L., F. y Ramírez Y., L. (2015). Prevalencia del síndrome de desgaste profesional en médicos residentes de un hospital de Mérida, Yucatán, México. Atención Familiar, 22(4), 111-114.

Ballinas A., G., Alarcón, C. y Balseiro C., L. (2009). Síndrome de burnout en enfermeras de un centro médico. Revista de Enfermería del Instituto Mexicano del Seguro Social, 17(1), 23-29.

Carlín, M. y Garcés E., J. (2010). El síndrome de burnout: evolución histórica desde el campo laboral al ámbito deportivo. Anales de Psicología, 26(1), 169-180.

Castillo I., Y., Orozco, J. y Alvis L., R. (2015). Síndrome de burnout en el personal médico de una institución prestadora de servicios de salud de Cartagena de Indias. Salud UIS, 47(2), 187-192.

Chorot, P. y Sandín, B. (1987). Escala de estrategias de coping (EEC). Madrid: Universidad Nacional de Educación a Distancia (Policopiado).

De la Rosa, G., Chang, S., Delgado, L., Oliveros, L., Murillo, D., Ortiz, R., Vela, G. y Yhuri, N. (2015). Niveles de estrés y formas de afrontamiento en estudiantes de medicina en comparación con estudiantes de otras escuelas. Gaceta Médica de México, $151,443-449$.

Díaz M., A. (2010). Burnout: un fenómeno creciente. Psicología desde el Caribe, 26, VIII-X.

Dickinson M., E., González, C., Fernández M., A., Palomeque R., P., González, E. y Hernández, I. (2007). Burnout syndrome among Mexican primary care physicians. Archivos en Medicina Familiar, 9(2), 75-79.

Feitosa S., K., Sousa L., A., Alves F., S., Gomes de C., P. y Mendes de A., L. (2016). Síndrome de agotamiento laboral (burnout) y sus representaciones entre profesionales de la salud. Investigación en Enfermería. Imagen y Desarrollo, 18(2), 137-152.

Galán, S. y Camacho E., J. (2012). Estrés y salud. Investigación básica y aplicada. México: El Manual Moderno.

González M., T. y Landero, R. (2007). Cuestionario de Afrontamiento del Estrés (CAE): validación en una muestra mexicana. Revista de Psicopatología y Psicología Clínica, 12(2), 189-198.

Guerrero B., E. y Vicente C., F. (2001). Síndrome de burnout o desgaste profesional y afrontamiento del estrés en el profesorado. Cáceres (España): Universidad de Extremadura.

Lazarus, R. y Folkman, S. (1984). Stress, appraisal, and coping. New York: Springer.

Manassero, M.A., Vázquez, A., Ferrer, V.A., Fornés, J. y Fernández, M.C. (2003). Estrés y burnout en la enseñanza. Palma de Mallorca (España): Universitat des Illes Balears. 
Martín M., J., Hernández B., M., Arnillas M., H. y García, M. (2009). Burn-out en el hospital: ¿estamos quemados los médicos? Medicina Balear, 24(3), 29-33.

Maslach, C., Jackson, S.E. y Leiter, M.P. (1996). The Maslach Burnout Inventory Manual. Palo Alto, CA: Consulting Psychologyst Press.

Meda L., R.M., Moreno J., B., Rodríguez, A., Morante, M.E. y Ortiz, G.R. (2008). Análisis factorial confirmatorio del MIB-HSS en una muestra de psicólogos mexicanos. Psicología y Salud, 18(1), 107-116.

Moreno, B., Seminotti, R., Garrosa, E., Rodríguez, R. y Morante, M.E. (2005). El burnout médico: la ansiedad y los procesos de afrontamiento como factores intervinientes. Ansiedad y Estrés, 11(1), 87-100.

Paris, L. y Omar, A. (2008). Predictores de salud laboral en médicos y enfermeros. Estudios de Psicología, 13(3), 233-244.

Parrello, S., Ambrosetti, A., Iorio, I. y Castelli, L. (2019). School burnout, relational, and organizational factors. Frontiers in Psychology, 10, 1695. Doi: 10.3389/fpsyg.2019.01695.

Pereda, L., Márquez F., G., Hoyos M., T. y Yáñez M., I. (2009). Síndrome de burnout en médicos y personal paramédico. Salud Mental, 32, 399-404.

Piñeiro, M. (2013). Estrés y factores relacionados en el personal sanitario de hospitalización psiquiátrica: un estudio de prevalencia. Enfermería Global, 31, 125-150.

Pistelli, Y., Perochena, J., Moscoloni, N. y Tarrés, M.C. (2011). Síndrome de desgaste profesional en médicos pediatras. Análisis bivariado y multivariado. Archivos Argentinos de Pediatría, 109(2), 129-134.

Rodríguez, A., Zarco, V. y Gonzáles, J.M. (2009). Psicología del trabajo. Madrid: Pirámide.

Sandín, B. y Chorot, P. (2003). Cuestionario de afrontamiento del estrés (CAE): desarrollo y validación preliminar. Revista de Psicopatología y Psicología Clínica, 8(1), 39-54.

Shaughnessy, J.J., Zechmeister, E.B. y Zechmeister, J.S (2007). Métodos de investigación en Psicología. México: McGraw-Hill.

Weber, A. y Jaekel, A. (2000). Burnout syndrome: a disease of modern societies? Occupational Medicine, 50(7), 512-517.

Instituto de Investigaciones Psicológicas - Universidad Veracruzana

ISSN impreso: 1405-1109
Psicología y Salud, Vol. 31, Núm. 2: 267-274, julio-diciembre de 2021 https://doi.org/10.25009/pys.v31i2.2695 